\title{
Analysis of the role of autopsy teaching in the context of precision medicine
}

\author{
Yan Fan ${ }^{1, a}$, Chengan Feng 2 ,b (Corresponding author) \\ ${ }^{1}$ Department of Anatomy and Histology \& Embryology, School of Basic Medical Science, Kunming Medical University \\ ${ }^{2}$ Department of Anatomy and Histology \& Embryology, School of Basic Medical Science, Kunming Medical University
}

\begin{abstract}
Autopsy is the foundation of general anatomy teaching and learning, and an indispensable part of medical education, and anatomy is the cornerstone of medical courses. Autopsy is an indispensable and important way to improve the quality of anatomy teaching. Nowadays, with the application of computer technology, the rapid development of molecular biomedicine and the emergence of other reasons, the status and function of autopsy in anatomy teaching have changed to varying degrees. Over the years, the reform of autopsy teaching has been continuously developed and updated. How to improve the effectiveness of autopsy teaching is an important course that requires us to study carefully. In the information age, autopsy teaching is affected by modern teaching techniques and methods. In addition, the source of corpses is very scarce and difficult to preserve. Most colleges and universities gradually adopt different teaching methods to replace autopsy teaching. In the context of precision medicine, autopsy teaching is in an irreplaceable position. This article starts with the importance of autopsy in anatomy teaching, discusses the current situation and future development direction of autopsy in anatomy teaching, and provides necessary help for further deepening the reform of autopsy education and teaching.
\end{abstract}

\section{Introduction}

Precision medicine is a hot spot in current international medical research. According to the different characteristics of individuals, the disease is divided into different subgroups, and targeted prevention, diagnosis and treatment are carried out [1]. However, this policy has aroused great attention to the quality of future medical staff and patient safety. However, precision medicine is an emerging medical technology, so the current national policy of precision medicine does not have a clear governance implementation plan, which has caused many universities to have a less clear attitude towards the opening of the subject of anatomy, I don't know how to implement this choice [2]. Compared with other disciplines, anatomy pays more attention to actual hand operation. The autopsy teaching method can be said to be an important method to complete the teaching of anatomy, and it can also give medical students a more detailed introduction, and autopsy allows medical students to observe and discover the mysteries of the human body at close range, so any other teaching methods cannot to truly meet the needs of anatomy teaching, it cannot achieve the expected goal of anatomy teaching. Therefore, autopsy is of great significance in anatomy teaching [3].

Autopsy is abbreviated as postmortem examination, which is to identify and determine various pathological changes through the observation of the body surface of the corpse, the naked eye of the internal organs and the morphological examination of the microscope, and combine the clinical manifestations of the deceased before his death, laboratory examinations, and diagnosis and treatment processes, etc. The examination method to clarify the nature of the disease and the cause of death is a basic research method of pathology [4]. The actual operation of autopsy can also give students the opportunity to fully understand the human body and deepen the understanding of the relationship between the morphological structure and direction of each organ [5]. This not only consolidates the theoretical knowledge of anatomy, increases the interest and enthusiasm of students, but more importantly, provides students with opportunities for surgical instruments, training their surgery, observation and independent thinking and problem-solving abilities will lay the foundation for clinical work[6]. Autopsy can generally be divided into: general anatomy, forensic anatomy, and pathological anatomy. The purpose of autopsy has three main points: (1) confirm the diagnosis, find out the cause of death, and improve the level of clinical medical treatment; (2) discover infectious diseases and new diseases in time; (3) accumulate data and specimens for scientific research and teaching [7 ].

Engels once said: "There is no medicine without anatomy" [8]. The questionnaire survey by Pete et al. clearly shows that $71 \%$ of people believe that anatomy is the most basic and important teaching method in anatomy teaching [9]. At present, many medical schools

\footnotetext{
afengca@126.com, ${ }^{b}$ fengca@126.com
} 
at home and abroad have gradually or completely abandoned anatomy teaching. In order to enable major universities to better carry out the courses in this area, two measures can be taken as follows. First, some existing problems are reported to the college and related departments through various channels, so as to strive for more attention and policies from the college and related departments. With support from other aspects, special cases and special cases can be realized; Second, retired technicians and professors with rich experience in autopsy are regularly invited to teach by passing, helping, and leading [10]. At the same time, if funding permits, the operator will be subsidized to attend the pathology academic conference, so as to expand the field of vision and knowledge, and to understand new technologies and equipment. But in fact, the anatomy teaching model can enable students to master the complex relationship between new professional terms and human body structure. During the autopsy, students can discuss in groups, which also helps to develop communication skills among student groups.

\section{Method}

\subsection{Consolidate the theoretical knowledge of anatomy and improve the practical ability}

The practice of autopsy can also give medical students an opportunity to fully understand the real human body, and deepen their understanding of the shape, structure and orientation of each organ. The demonstration is not a mechanical understanding of the structure, but the deeper meaning is to inspire students to think, link the structure and function of the human body, and explain the function on the spot, so that the students can deepen the understanding and memory of the human structure. For medical students to form an accurate concept, the posture of autopsy is different, and the difference must be clearly pointed out. When emphasizing specific operations, it should be emphasized that no matter how the specimen, model or patient is placed, the detailed location of each organ and structure should be described according to the anatomical posture. Teaching teachers should first use themselves to demonstrate, and then every student participates in it. Teachers visit and guide and correct students' mistakes and puzzles at any time. They not only play the leading role of the instructor, but also play the main operation role of the students, which not only enhances the awareness of the medical students to participate in the actual operation. The instructor's key introduction and accurate description of the location of the anatomy will help students form the concept of accurate autopsy operation.

\subsection{Strengthen medical ethics education}

The remains of the deceased donor became the first step for medical students to enter the medical world. Those donors are the noblest, the most selfless, and the most respectable. Their selfless dedication has benefited medical students for life. Before performing an autopsy, medical students should express their respect to the donor in some ways, such as holding a simple silent mourning ceremony, keeping the operation process serious, and after the body is used up, a memorial ceremony including the family of the deceased will be held. Respect, sympathize and protect the body through zero-distance contact with the body. These ceremonies are also the teaching part of academic education. To sum up, autopsy is still the main teaching method of human anatomy in the future, but teaching based on autopsy alone cannot meet all the needs of current medical courses, and must be supplemented by updated and advanced teaching methods or learning methods. This interdependence between different teaching methods and autopsy constitutes a new type of teaching mode, which may be the future development direction of anatomy teaching.

\subsection{Increasing inter-professional education and providing opportunities for real learning}

Team-based autopsy activities have also been used to explore the field of interprofessional education (IPE). The implementation of interprofessional education is related to the development of students' cooperative behavior. In other words, inter-professional education helps promote the development of collaborative skills in the workplace, thereby ensuring that clinical errors are reduced and patient treatment outcomes are improved. In the research of the interdisciplinary autopsy course. Autopsy can promote cross-professional learning of medical students. These are all important topics in medical education. Anatomy educators all believe that autopsy teaching is superior to other teaching methods for anatomy teaching. They agree that autopsy teaching helps students to learn anatomy and develop their own skills during clinical work in the future. Autopsy teaching provides medical students with the opportunity to receive "real learning" on a regular basis. Its comprehensive learning model includes teaching methods such as autopsy, pathology, clinical evidence, imaging, etc., which provides strong support for "real learning".

\section{Experiment}

\subsection{Subject}

A total of 50 fresh autopsy specimens were collected, including 5 fetuses, 7 newborns and 38 adults for autopsy. The youngest is 30 weeks old, and the oldest is 91 years old. Collect heart, lung, liver, spleen, kidney and brain tissue from each autopsy specimen according to the needs of autopsy. And remove the umbilical cord tissue from the fetal specimen. 12 specimens of fetus and newborn; there are also 38 specimens of adult cadavers. In order to study the operation of autopsy and show the specific theories of the autopsy course for medical students, the teacher led the students to conduct experiments in groups of these 50 autopsy specimens, 
personally perform the autopsy operation, and observe and experience the process at close range. In the process of anatomy, the leading teacher needs to explain each step in detail, and introduce the structure of the human body and the functions and medical terms of each part of the human body in detail. The heart, lung, liver, spleen, kidney, and brain tissues of these 50 cadavers were dissected. These tissues need to be explained in detail, and then experiments are carried out, and the instructional experiment is personally conducted to stain CD105, so as to make the medical students' practical ability Get promoted, and finally lead medical students to complete this experiment.

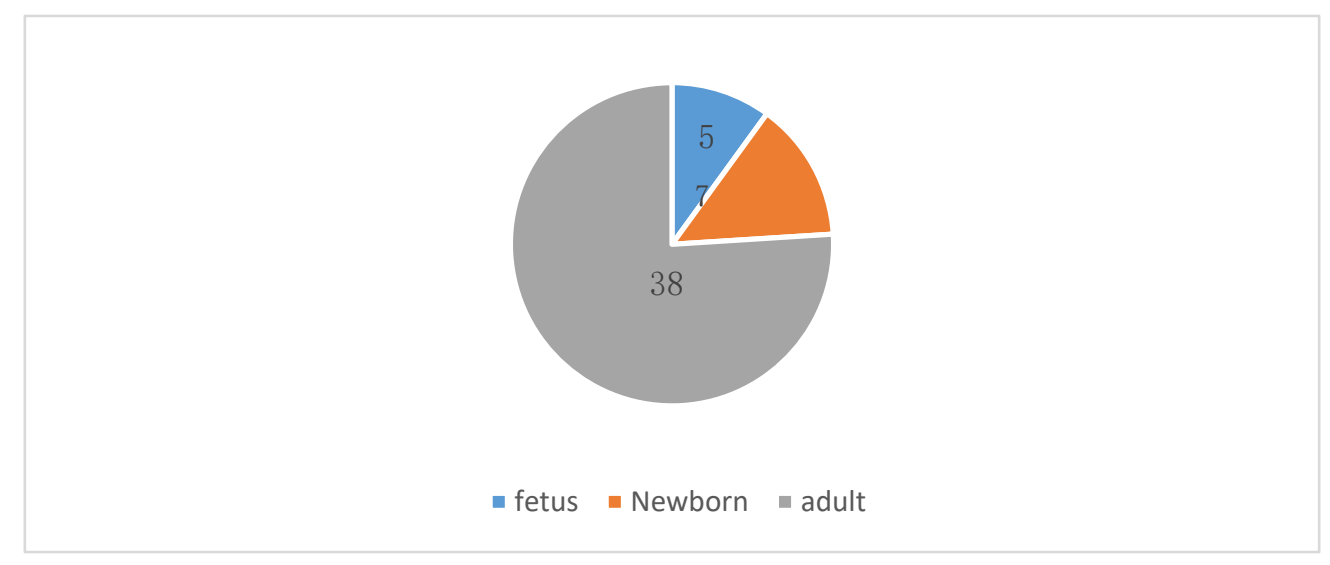

Figure 1. Survey sample distribution

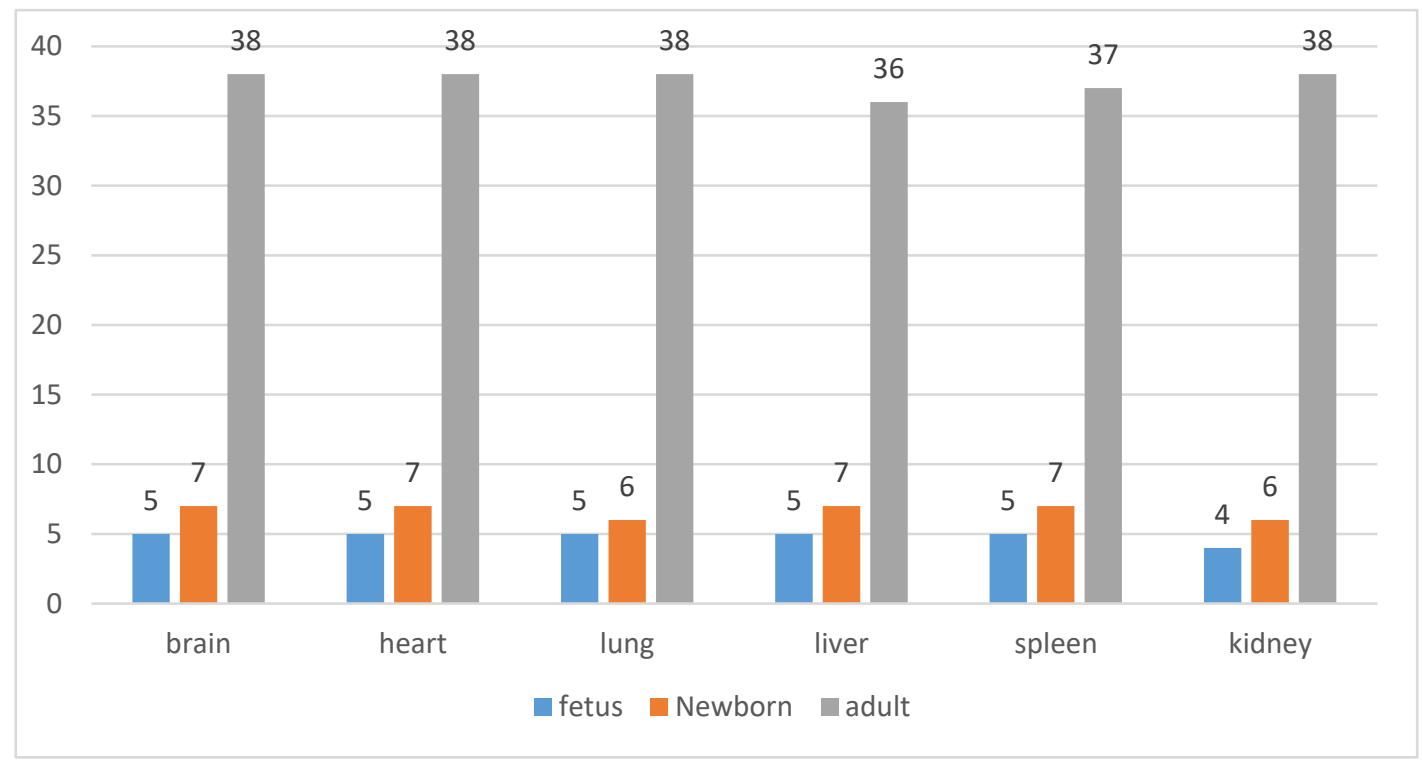

Figure 2. Number of tissue samples dissected

\subsection{Experimental method}

(1) Quantitative analysis method: This article collects a large amount of data through WTO, UNCTAD, WORLDBANK and other databases, and conducts a quantitative analysis of Chinese and international autopsy teaching systems, and provides a basis for related research.

(2) Analytical method: This paper analyzes the collected data to find the correlation between the role of precision medicine and autopsy teaching in my country, and uses standard analysis to evaluate the role of autopsy teaching in the context of precision medicine.

(3) Result comparison method: This article studied the anti-human CD 105 Mab E9 staining results by dissecting the above 50 autopsy cases and taking out the dissected samples to observe and compare the content of anti-human CD 105 Mab E9 in different organs in the human body.

\section{Results}

\subsection{Investigation and analysis of experimental data}

In 50 frozen sections stained with CD105, Mab-E9 positive cells were mainly distributed in the EC cell membrane and cytoplasm, and the cell membrane was stained more than the cytoplasm. Collect heart, lung, liver, spleen, kidney and brain tissues from each anatomical specimen, and extract umbilical cord tissue from fetal 
specimens. When collecting specimens, make sure to perform an autopsy within 48 hours after death, and prepare materials, or put the specimens in a sterile cryotube for storage in liquid nitrogen within 2 hours. All tissue specimens were washed with $0.01 \mathrm{M}$ PBS, and then made 5 7 8um continuous frozen sections on a thermostat. In the brain, heart, liver, spleen, lung and kidney, the positive staining of CD34 is very weak, and only some capillaries, arterioles and veins in the matrix are weakly stained. In the brain, heart, liver, spleen, lung and kidney, some capillary strips and small arteries and veins were positively stained. Table 1 and Table 2 show the results of CD105 positive expression in 50 autopsy specimens.

Table 1. CD105 staining results of brain, heart, lung, liver, spleen and kidney tissues of 12 fetuses and newborns

\begin{tabular}{|c|c|c|c|c|c|c|}
\hline Group & \multicolumn{3}{|c|}{ Capillaries } & \multicolumn{3}{c|}{ Small blood vessels } \\
\hline & + & \pm & - & + & \pm & - \\
\hline brain & 9 & 0 & 0 & 7 & 0 & 3 \\
\hline heart & 7 & 1 & 0 & 5 & 1 & 5 \\
\hline liver & 5 & 1 & 2 & 2 & 2 & 5 \\
\hline spleen & 6 & 1 & 1 & 3 & 2 & 4 \\
\hline lung & 6 & 3 & 0 & 2 & 2 & 4 \\
\hline kidney & 7 & 2 & 0 & 4 & 1 & 5 \\
\hline
\end{tabular}

Table 2. CD105 staining results of 38 adult brain, heart, lung, liver, spleen and kidney tissues

\begin{tabular}{|c|c|c|c|c|c|c|}
\hline Group & \multicolumn{5}{|c|}{ Capillaries } & \multicolumn{3}{c|}{ Small blood vessels } \\
\hline & + & \pm & - & + & \pm & - \\
\hline brain & 29 & 0 & 0 & 27 & 0 & 3 \\
\hline heart & 34 & 1 & 3 & 25 & 1 & 15 \\
\hline liver & 15 & 15 & 2 & 12 & 2 & 25 \\
\hline spleen & 26 & 1 & 1 & 13 & 2 & 24 \\
\hline lung & 26 & 3 & 2 & 15 & 2 & 24 \\
\hline kidney & 17 & 4 & 12 & 14 & 1 & 19 \\
\hline
\end{tabular}

It can be seen from the above table that the expression of CD105 monoclonal antibody E9 on capillaries and small blood vessels during the preparation of viscera specimens is significantly different from the row $\times$ list test. The actual results showed that in the brain, heart, liver, spleen, lung and kidney tissues, the EC positive rate of CD105-labeled capillaries were better than $\mathrm{CD} 34$, but there was no significant difference between CD105 and factor VIII-labeled EC positive rates. The EC difference of blood vessels in other organs is statistically significant. However, there are significant differences between CD34 and factor VIII markers, and there is no significant difference in the expression of EC in other organs. CD34 staining of liver and spleen tissues is very weak, sinusoidal EC is slightly stained, only a few interstitial capillaries and small arteries and veins are stained and lightly stained; a few capillaries in various tissues of brain, heart, liver, spleen, lung and kidney The staining results of umbilical cord tissues were similar to CD1005 monoclonal antibody E9 staining results.

\section{Conclusion}

The development of anatomy has gone through centuries. Long-term medical practice has proved that the existence of an autopsy is necessary. Even if medical students do not perform autopsy surgery, even if they receive more advanced technical training, it is difficult for medical students to perform surgery, examination and treatment in the clinic. For students who are new to medicine, learning this course is essential. Anatomy is a necessary teaching method to improve the quality of anatomy learning. Autopsy not only allows medical students to truly understand the structure and organization of the human body,but also consolidates the theoretical knowledge learned in class and in books.In addition it can also be used for actual operations in the above class or demonstrations by the teacher to mobilize medical students to teach anatomy. Interest can cultivate cultivate students' independent thinking and independent hands-on ability in actual operation and later clinical surgery. We also need to gradually explore more effective solutions to make anatomy teaching popular. Scientific experiments 
have shown that in the speed of information reception and processing, the visual center is significantly faster than the auditory center, with a speed difference of more than 500 times. Three quarters of the human brain is used for vision. In fact, it uses the "brain" to observe the world through visual images. The ability of the right brain to remember graphics is much higher than that of the left brain. Once you remember this number, it is difficult to forget it, even if you forget it, it is easy to remember it after reading it. Using the sensitive characteristics of the human brain to graphics, students can observe more specimens and graphics. When reviewing, they will reproduce specimens and characters in their minds, that is, use image thinking to deepen their memory. In short, autopsy is of great significance to anatomy education and teaching, and will play an important role in the development of the future medical profession.

foundation projects: The school-level first-class course project of Kunming Medical University in 2020 (Project number: 2020JXZ011)

foundation projects: The school-level teaching research and reform project of Kunming Medical University ( Project number: 2020-JY-Y-008; 2020-JY-Y-011)

foundation projects: The school-level quality project of Kunming Medical University in 2019 - the construction of Human Anatomy blended curriculum

\section{References}

1. Loscalzo J . Precision Medicine[J]. Circulation Research, 2019, 124(7):987-989.

2. Kosorok M R, Laber E B . Precision Medicine[J]. Annual Review of Statistics \& Its Application, 2019, 6(1):263-286.

3. Aleh K . Analysis of mortality in necrotizing pancreatitis according to autopsy data[J]. Pancreatology, 2018, 18(4):S101-S102.

4. Chaudhary B L, Singh R K, Singh S, et al. Recent trends of medical negligence - An autopsy based study at lady hardinge medical college, New Delhi[J]. IP International Journal of Forensic Medicine and Toxicological ences, 2020, 5(2):35-38.

5. Hasmi A H, Subramaniam K, Lai P S, et al. Adulteration in the Drug of Abuse: An Autopsy Study on the Prevalence and its Role in Natural Death $[\mathrm{J}]$. Toxicology International, 2019, 25(2):124-129.

6. Fred H L , Gonzalo J D . Reframing Medical Education[J]. Texas Heart Institute journal / from the Texas Heart Institute of St. Luke's Episcopal Hospital, Texas Children's Hospital, 2018, 45(3):123-125.
7. Mladenovic J , O'Brien M C , Hoppman R . Shaping the Future of Point-of-Care-Ultrasound in Medical Education[J]. Southern medical journal, 2018, 111(7):449-451.

8. Ruban A , Sankaran P S . A proposal based on a review of reforms for improving medical education in India[J]. Postgraduate Medical Journal, 2018, 94(1109):187-188.

9. Yasuda J K . Response to Henry Thomson's Review of On Feeding the Masses: An Anatomy of Regulatory Failure in China $[\mathrm{J}]$. Perspectives on Politics, 2020, 18(2):570-570.

10. Bayindir N , Kinci N . ALTHENIA ORIENTALIS (POTAMOGETONACEAE) IN TURKEY: HABITAT CONDITIONS, MORPHOLOGY AND ANATOMY[J]. Communications Faculty of ence University of Ankara Series C Biology Geological Engineering and Geophysical Engineering, 2020, 29(2):243-253. 